\title{
A Utilização de Ferramentas Tecnológicas no Ensino e Aprendizagem em Matemática para Alunos com Transtorno do Espectro Autista
}

\author{
Anderson Daniel Stochero ${ }^{1}$, Bruna Willig Kopplin ${ }^{1}$, Samuel Muller Forrati ${ }^{1}$, \\ Andréa Pereira ${ }^{1}$, Cristiane da Silva Stamberg ${ }^{1}$ \\ ${ }^{1}$ Instituto Federal de Educação, Ciência e Tecnologia Farroupilha - IFFAR (campus \\ Santo Ângelo) CEP 98806-700 - Santo Ângelo - RS - Brazil \\ anderson_stochero@yahoo.com.br, \\ \{brunawkopplin, samuel. forrati\}@gmail.com, \\ \{andrea.pereira, cristiane.stamberg\} @iffarroupilha.edu.br
}

\begin{abstract}
This study questions the use of technological tools in teaching and learning mathematics for students with disabilities. In this sense, the need to implement the use of technological tools in education requires a rethinking of pedagogical practice with a resizing of the school that consists not only of acceptance, but also of valuing differences. The study is in the phase of implementation and analysis of the most relevant factors about the characteristics of the autistic subject, through interviews with professionals in the field, educators, associations and the research subjects themselves.
\end{abstract}

Resumo. Este estudo problematiza o uso de ferramentas tecnológicas no ensino e aprendizagem da matemática para alunos com deficiência. Nesse sentido, a necessidade de implementar o uso de ferramentas tecnológicas na educação requer um repensar da prática pedagógica, com um redimensionamento da escola que consiste não somente na aceitação, mas também na valorização das diferenças. $O$ estudo encontra-se em fase de implementação e análise dos fatores mais relevantes acerca das características do sujeito autista, através de entrevistas com profissionais da área, educadores, associações e os próprios sujeitos da pesquisa.

\section{Introdução}

A inclusão de pessoas com deficiência na sociedade não requer apenas acessibilidade de locomoção. Requer, também, aceitação social e a garantia de que ela ocorra. A criança, ao ser diagnosticada com qualquer tipo de deficiência, seja ela intelectual, física ou auditiva, tem direitos semelhantes às demais. Além disso, o cuidado especial destinado a ela, principalmente no que diz respeito à educação, é um dever do Estado e um direito previsto em lei, conforme Guia de direitos (2017).

É importante ressaltar que não basta ter o acolhimento, uma vez que um aluno com deficiência necessita de condições efetivas de aprendizagem e desenvolvimento de suas potencialidades. Dessa maneira, é necessário, que os sistemas de ensino se organizem para assegurar essas matrículas e também a permanência de todos os alunos, sem perder de vista a intencionalidade pedagógica e a qualidade do ensino, ideia corroborada por Frias e Menezes (2008). Para garantir a assimilação de conceitos, a 
VI Congresso Brasileiro de Informática na Educação (CBIE 2017)

Anais do XXIII Workshop de Informática na Escola (WIE 2017)

criança que possui deficiência terá direito de participar do desenvolvimento de atividades tanto na educação regular, quanto em escolas de educação especial. A escolha do tipo de educação a ela destinada possui aparato legal e deve ser pautada a partir da filosofia que a instituição de ensino deseja desenvolver, conforme aponta o Guia de direitos (2017).

Diante desse contexto social a inclusão tornou-se imprescindível, e necessária de ser estudada e trabalhada dentro e fora das instituições de ensino pois as interações sociais, importantes para o desenvolvimento da criança, servem de estímulo a aprendizagem tanto em sala de aula, como, para a formação da sociedade atual que seja ativa e solidária. Sendo assim, este artigo tem como objetivo apresentar as ações desenvolvidas a partir de um projeto de pesquisa que possui como proposta encontrar caminhos para a utilização de ferramentas tecnológicas, através da implementação de jogos, capazes de contribuir com processo de ensino e aprendizagem da matemática para alunos com transtorno do espectro autista, possibilitando a promoção de aprendizagens significativas para um maior número possível de pessoas no ambiente escolar.

Entre os vários conhecimentos que a criança constrói e adquire durante a sua formação acadêmica, a matemática ocupa um lugar de destaque em sua vida. Isto pode estar relacionado com a importância da matemática que pode ser observada na conquista de grandes avanços tecnológicos e no embasamento teórico de muitas profissões de acordo com Carvalho et al (2016).

Os desafios do ensino de matemática para pessoas com deficiência é um dos temas de extrema importância nas escolas uma vez que a abordagem deve ser diferenciada. Diante disso, para o aprendizado desse componente curricular e para a identificação das tecnológicas mais adequadas para o desenvolvimento desse projeto, estão sendo realizados estudos, para a criação de jogos que permitam o aprendizado com significado, permitindo a inclusão e também a integração de crianças autistas no ambiente educacional. O conjunto desses dados, associados à fundamentação teórica permite reflexões e análises das percepções e ações a serem assumidas, apropriando-se das dificuldades, barreiras, trabalhos bem-sucedidos, e desenvolvidos pelas escolas que possuem alunos com alguma deficiência.

\section{Contextualização}

Com o aumento exponencial do uso de tecnologia em todas as idades, o acesso a informações se tornou muito fácil, surgindo novas formas de agir e pensar. Santos et al (2015) afirmam que um profissional capaz de se reinventar de acordo com as necessidades do momento se tornou mais desejado do que um profissional que sempre age da mesma forma, que nunca está disposto a mudar. Diante disso, ao longo do processo de ensino e aprendizagem, os educadores sentem à necessidade de buscar aporte das tecnologias da informação como estratégias metodológicas de ensino.

Os jovens têm mais facilidade de lidar com a linguagem dos meios digitais do que a linguagem escrita, afirmam Sampaio e Leite (2004). Para o autor, os jovens se identificam com os meios eletrônicos por carregarem características semelhantes, dentre elas, a constante presença de informações visuais e o rápido processamento de múltiplos recursos simultâneos. As Tecnologias da Informação e Comunicação (TIC) têm uma influência direta na aquisição de competências e no desenvolvimento das 
aprendizagens. São estimulantes tanto pela variedade de recursos quanto pela forma como se apresentam pois tem oportunizado às pessoas maneiras cada vez mais dinâmicas e atrativas de comunicação e interação social. De acordo com Menezes e Roza (2016), aos poucos os indivíduos estão abandonando sua condição passiva diante de atividades cotidianas, otimizando-as e assim tornando-se sujeitos ativos, com facilidade e em um curto espaço de tempo, integrando as novas tecnologias a sua rotina, através da utilização de celulares, tablets ou computadores.

Todavia, não basta inserir hardwares e softwares no ambiente educacional, é de suma importância que a escola reflita acerca de sua utilização e na promoção significativa de aprendizagem de modo que o sujeito seja capaz de desenvolver e executar algo de forma que a construção do conhecimento ocorra através da resolução de problemas e da comunicação. É necessário que os professores saibam lidar e desenvolver o processo de ensino-aprendizagem com todos os alunos, sem exceções. Para isso, é oportuno possibilitar aos docentes a participação em cursos que discutam estratégias educacionais visando à participação ativa e consciente de todos os alunos no processo de ensino e aprendizagem. Esses cursos devem atender as necessidades de preparo que os professores têm para desenvolver práticas docentes realmente inclusivas, conforme aponta Frias e Menezes (2008).

Nesta perspectiva, legitima-se o desenvolvimento desse projeto de pesquisa, pois há uma intencionalidade em proporcionar ao aluno novas experiências de ensino e aprendizagem e também promover a inclusão de jovens no ambiente educacional, diante do reconhecimento da importância na utilização da tecnologia nesse processo, trabalhando com o desenvolvimento jogos e materiais didáticos voltados para indivíduos com Transtorno do Espectro Autista (TEA). As TICs apresentam uma possibilidade de inovação dos recursos, numa tentativa de melhorar o desenvolvimento desses sujeitos.

De acordo com Bosa (2016) o autismo pode ser classificado como um transtorno invasivo do desenvolvimento e abarca algumas dificuldades ao longo da vida do portador afetando as habilidades sociais e comunicativas além de comportamento e interesses limitados e repetitivos. De forma geral, a maioria dos jovens autistas apresentam dificuldades de compreensão de linguagem abstrata ou dificuldade de lidar com sequências complexas de instruções que necessitam ser decompostas em unidades menores. Sendo assim, para auxiliar no processo de inclusão e aprendizagem dos indivíduos autistas, é comum a utilização de softwares e hardwares para facilitar a assimilação do conhecimento, apesar de não haver grande quantidade softwares apropriados para o grupo em questão, de forma que atenda às necessidades baseando nas características desse transtorno do espectro autista. Contudo, nos últimos anos têm sido comprovada a eficiência da utilização de softwares e hardwares assistivos no desenvolvimento cognitivo e melhoria de habilidades de jovens autistas, afirmam Souza et al (2016).

Os recursos tecnológicos têm sido aplicados em sujeitos com TEA, o uso do computador e em especial de ambientes digitais de aprendizagem adaptados aos interesses e necessidades dos sujeitos mostram-se relevantes e importantes no desenvolvimento e interação das pessoas com autismo. Segundo Bittencourt e Francisco (2015), estudos propõem uma visão sobre os processos de interação que se estabelecem em ambientes digitais considerando o TEA, em que o uso desses ambientes mostrou-se 
VI Congresso Brasileiro de Informática na Educação (CBIE 2017)

Anais do XXIII Workshop de Informática na Escola (WIE 2017)

relevante no desenvolvimento da interação social de sujeitos com autismo. Para Passerino (2005) é necessário estabelecer estratégias para serem aplicadas em diferentes ambientes educativos de forma que a inserção da tecnologia possa acrescentar o diferencial qualitativo na promoção da interação social de sujeitos com autismo.

\section{Trabalhos relacionados}

Visando coletar informações de layout, conteúdo e abrangência, entre outras características a serem consideradas no desenvolvimento do jogo, foi conduzida uma pesquisa na internet de softwares matemáticos gratuitos que trabalham com as operações básicas. Entre eles podemos destacar os seguintes:

Spiko and the Math Masters: o jogo tem um personagem chamado Spiko que ajuda a criança a cumprir suas tarefas, dando soluções para problemas envolvendo soma, diminuição, multiplicação, divisão e fração, em Lemon (2017). São problemas simples, para crianças de 6 a 8 anos que ainda estão na etapa inicial do aprendizado matemático, através das quatro operações básicas. O programa é executado em inglês.

Sebran's ABC: é um jogo que ensina as crianças a ler e escrever. Contem vários tipos de exercícios diferentes, no caso do jogo matemático, introduz os números de 1 a 9 que são usados em exercícios de Somar, Subtrair e Multiplicar, cada um com dois níveis de dificuldade, em Seterra (2017). O programa é executado em diferentes idiomas, incluindo Português, Inglês, Francês, Espanhol e Alemão.

TuxMath: o jogo tem como personagem principal um Pinguim, que vai destruir meteoros que caem nas cidades com sua arma de raios laser, ativada pelas soluções matemáticas, em Tux (2017). Os meteoros são acompanhados por operações matemáticas e para destruí-los você terá que resolver os problemas.

Em comparação a esses jogos analisados, a proposta diferenciada do projeto é desenvolver um jogo com significado que vá além da relação superficial entre os algoritmos, mostrando que é possível refletir sobre as relações entre diferentes aspectos que envolvem as operações matemáticas, explicando os significados e as relações entre os termos. Pensar em uma aprendizagem significativa na matemática, requer ações de ensino direcionadas, que possibilitem aos alunos utilizar-se do pensamento concreto para posteriormente estabelecer o pensamento conceitual mais adequado. $\mathrm{O}$ projeto apresenta ainda como vantagens a não utilização da Internet para o desenvolvimento das atividades; e sua facilidade de uso, não exigindo um conhecimento tecnológico prévio por parte dos professores.

\section{Metodologia}

O desenvolvimento do projeto foi baseado inicialmente em referenciais bibliográficos, posteriormente submetido ao comitê de ética, para que se tornasse possível a continuidade da pesquisa.

A partir do parecer do comitê de ética, e dando sequência ao projeto, foram realizadas entrevistas com profissionais especializados e que atuam diretamente com os alunos autistas. Para as entrevistas, foi estabelecida a metodologia de entrevista semiestruturada, a qual compreende um roteiro com perguntas fechadas e abertas, permitindo maior flexibilidade nas conversas e entendimento nas questões trazidas pelo interlocutor. Minayo (2010) apresenta que esse modelo favorece a comunicação entre entrevistador e entrevistado possibilitando aprofundar as reflexões em torno de 
determinado tema. Os participantes das entrevistas, já atuam em uma perspectiva de inclusão, pois são profissionais da educação e gestores, articulados com o uso da tecnologia no ensino e aprendizagem de matemática, com intuito de conhecer a realidade e as necessidades no processo de ensinar e aprender do sujeito com autismo. As instituições visitadas e entrevistadas no primeiro momento foram: Associação de Pais e Amigos do Autista e Centro de Atenção Psicossocial Infância e Adolescência (CAPS II Hakani).

Nesse mesmo espaço de tempo, foi realizado um levantamento de softwares matemáticos livres disponíveis que auxiliassem o ensino e aprendizagem em matemática. Após o levantamento desses softwares e a realização das entrevistas, realizou-se mais uma etapa do estudo, que foi a aplicação dos softwares matemáticos pesquisados, com participação de quatro alunos com TEA. Os jogos foram previamente instalados em todas as máquinas do laboratório de informática do CAPS II, deixando-os pronto para a aplicação dos jogos.

Esse teste, de aplicação dos jogos em laboratório, realizado com os alunos com TEA consistiu em explicar as regras, objetivos e opções do jogo, sugerindo a realização das fases e observando o comportamento dos mesmos frente ao jogo. Posteriormente, foi realizado com os alunos um diálogo sobre as facilidades e dificuldades, explicitando também quais eram suas preferências.

Após esse momento, o estudo buscou, a partir das observações feitas, explorar os softwares, inserindo também o material concreto para compreensão das operações matemáticas, como material dourado e palitos, com o objetivo de mostrar que as operações precisam estar sempre contextualizadas e para que os algoritmos não se tornem vazios de significado. Neste momento, o jogo encontra-se em fase de implementação com a preocupação de atender as necessidades dos alunos com TEA, e também incluindo interfaces com a finalidade de mostrar o domínio pleno das etapas permitindo que a ferramenta tecnológica possa permitir a compreensão e o porquê de cada procedimento.

\section{Resultados e Discussão}

Busca-se através deste estudo criar ferramentas aliadas a tecnologia, aos alunos com TEA, que possam possibilitar meios para compreender as operações matemáticas com eficiência. De acordo os Parâmetros Curriculares Nacionais,

[...] para desempenhá-lo, além de conhecer as condições socioculturais, expectativas e competência cognitiva dos alunos, precisará escolher o(s) problema(s) que possibilita(m) a construção de conceitos/procedimentos e alimentar o processo de resolução, sempre tendo em vista os objetivos a que se propõe atingir (BRASIL, 1997, p.40).

Assim, torna-se necessário levar em conta as experiências e atividades diárias dos alunos, buscando a contextualização do conteúdo a ser ensinado. Dessa maneira, para o desenvolvimento do estudo, o contato inicial se estabeleceu com um profissional fisioterapeuta que possui estudos sobre o autismo. A entrevista possibilitou expandir os conhecimentos sobre as principais características dos jovens autistas, possibilitando uma reflexão mais aprofundada e norteando os passos a serem traçados para $o$ desenvolvimento do projeto.

Outro encontro relevante que merece destaque ocorreu na instituição CAPS II 
Hakani, onde os integrantes do projeto participaram de uma reunião com a coordenadora da instituição e mais cinco psicólogas que atendem crianças com autismo, objetivando expor o projeto em desenvolvimento e conhecer mais das características do público-alvo deste estudo. Após parecer do comitê de ética para o desenvolvimento da pesquisa, realizamos entrevistas com as psicólogas que, relataram os trabalhos e as atividades que realizam com as crianças e orientaram quanto ao nível de jogo a ser implementado, destacando que o mais adequado para se trabalhar matemática é com as etapas iniciais como, por exemplo: as quatro operações básicas. Foi agendado, também, um encontro no laboratório de informática da instituição, com crianças previamente selecionadas pelas psicólogas para mostrar alguns softwares existentes voltados ao ensino da matemática com a finalidade de analisar o comportamento e a aceitação das crianças quanto a aplicabilidade de softwares de jogos. Nesse encontro foi possível identificar quais as características mais relevantes no processo de aprendizado das crianças autistas, norteando a criação dos jogos com o auxílio de ferramentas tecnológicas, visando identificar as necessidades de cada um, seus gostos e peculiaridades. Essa fase nos permitiu avançar para as próximas etapas, que foram a seleção e construção de jogos e materiais didáticos com o auxílio de softwares e ferramentas tecnológicas, contribuindo para o processo de ensino e aprendizagem da matemática, componente curricular obrigatório na educação básica brasileira.

Quanto aos recursos a serem utilizados, busca-se através dos mesmos benefícios ao processo de ensino e aprendizagem desses alunos, em que a criação do software atenda às necessidades dos alunos com TEA, incluindo interfaces que levem estes a compreensão e o porquê de cada procedimento, em que o desenvolvimento permita ao mesmo desenvolver a capacidade de construir, de forma autônoma, o conhecimento sobre um determinado assunto, segundo Bona (2009).

Tal fato foi observado no uso dos softwares livres aplicados com os quatro alunos, em que percebeu-se o comportamento de empolgação dos alunos e poucas dificuldades em trabalhar com software após as instruções dadas. É válido dizer, que foi dada a possibilidade aos alunos de escolha dos níveis do jogo de acordo com o que mais interessava a cada um e os mesmos escolhiam os níveis de acordo com o grau de facilidade para resolver o algoritmo. Dessa forma o jogo proporcionou aos alunos a possibilidade de acompanhar os diferentes níveis de aprendizagem. Ressalta-se que mesmo surgindo dúvidas, os alunos não paravam a realização da atividade, ficando concentrados até o final.

Outro fato observado foi o comportamento dos alunos durante as atividades, em que foi verificado atitudes de entusiasmo resolvendo problemas matemáticos através de um recurso mais atraente e ao mesmo tempo divertido, tornando a matemática mais interessante e dinâmica, inclusive usando para a criação do software o que os alunos mais gostam, como: resolver operações matemáticas usando animais, veículos, alfabeto, frutas, conforme as interfaces criadas.

A seguir, são apresentados alguns exemplos iniciais das telas que irão compor o jogo. Para a criação das imagens foram utilizados elementos gráficos do portal livre Freepik (2017) e adaptadas para o contexto do jogo proposto utilizando ferramentas de edição gráfica como Inkscape (2017) e LibreOffice Impress (2017).

O Freepik é um site onde é possível encontrar mais de um milhão de arquivos gráficos de gratuitos para download, desde vetores, ilustrações, ícones, fundos, temas 
VI Congresso Brasileiro de Informática na Educação (CBIE 2017)

Anais do XXIII Workshop de Informática na Escola (WIE 2017)

HTML e fotos gratuitas, com licença Creative Commons, seguindo as últimas tendências em design para serem utilizadas em sites, blogs, banners, apresentações e muitas outras aplicações. Já o Inkspace é um software profissional de edição e criação de imagens vetoriais e é uma alternativa livre e gratuita ao Adobe Illustrator (2017) e ao CorelDraw (2017). E, o LibreOffice Impress, é uma ferramenta gratuita poderosa para a criação de apresentações multimídias, que vem acompanhada da suíte de aplicativos para escritório LibreOffice, suas ferramentas podem ser comparadas a outros softwares pagos na área, como o Microsoft Office PowerPoint (2017), por exemplo.

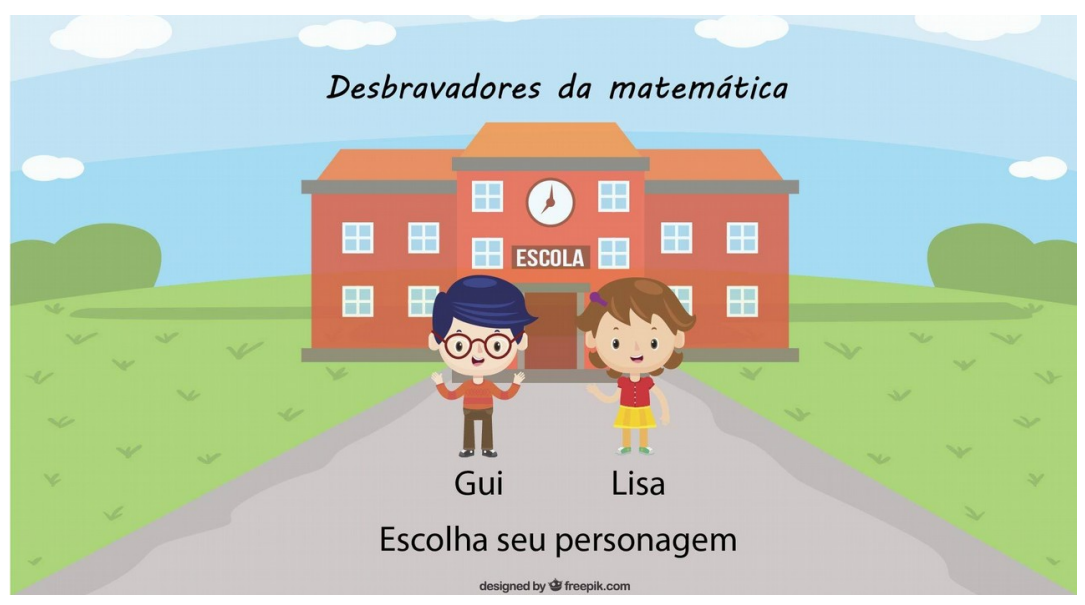

Figura 1: Protótipo da tela inicial do jogo - escolha dos personagens

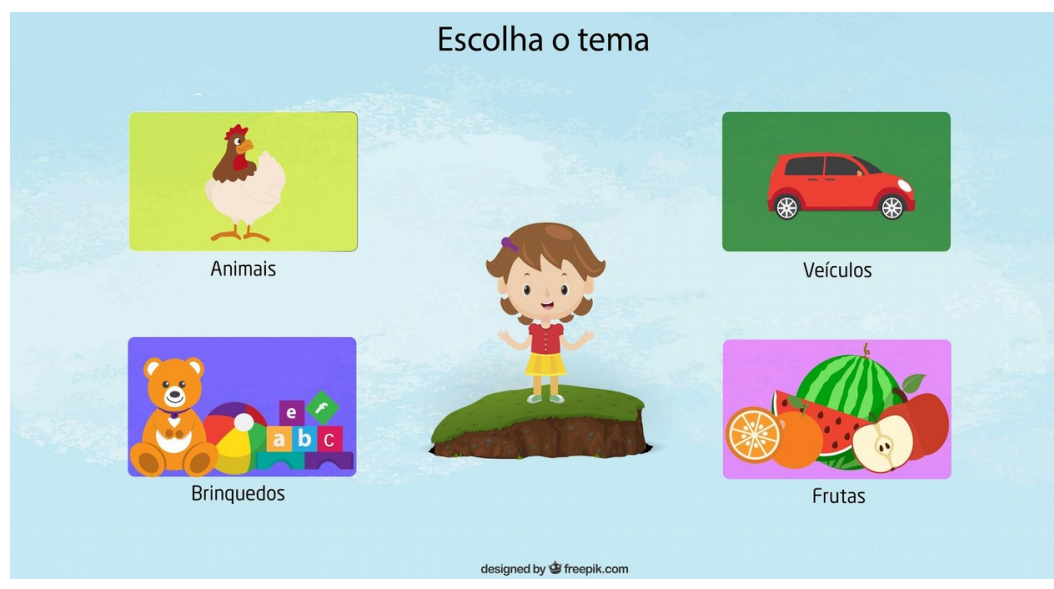

Figura 2: Protótipo da segunda tela do jogo - escolha da tela após a personagem "Lisa" ser escolhida 
VI Congresso Brasileiro de Informática na Educação (CBIE 2017)

Anais do XXIII Workshop de Informática na Escola (WIE 2017)

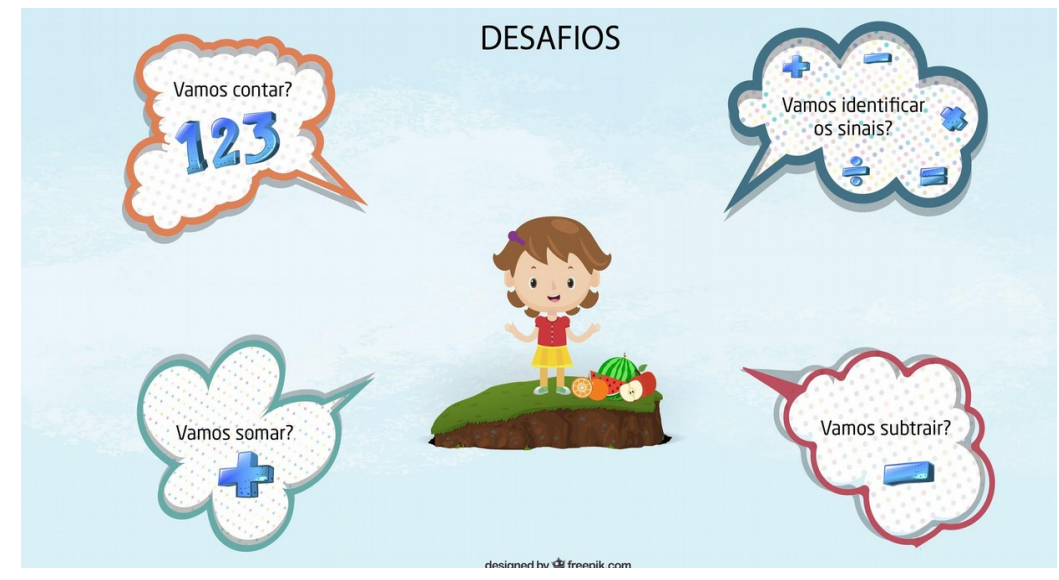

Figura 3: Protótipo da terceira tela do jogo - após selecionar o tema "frutas"

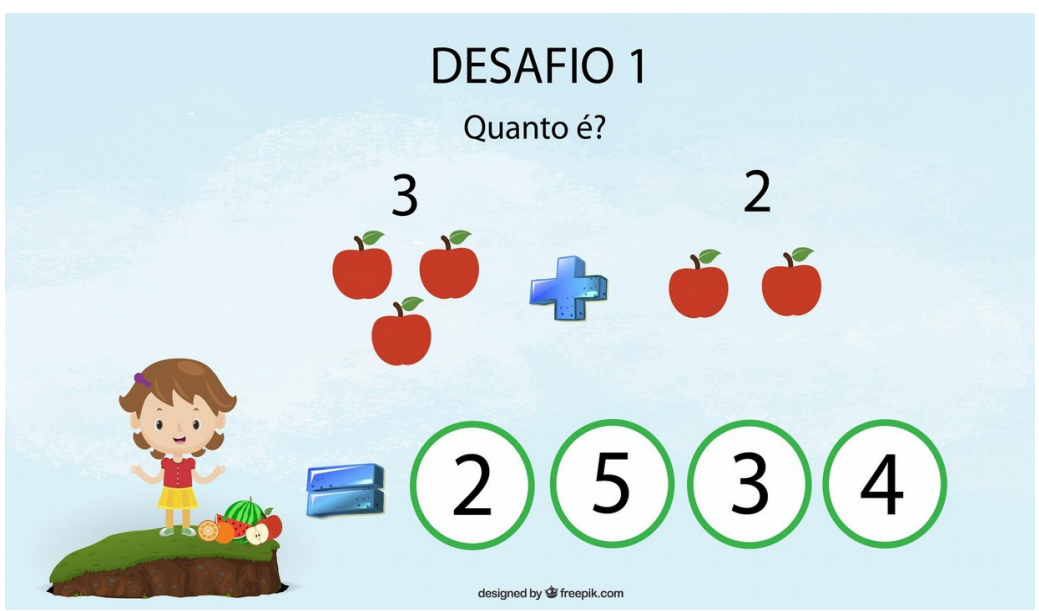

Figura 4: Protótipo do desafio "soma" proposto pelo jogo - tema "frutas"

\section{Considerações finais}

A partir do desenvolvimento do projeto espera-se como resultado a efetiva melhoria no processo de ensino e aprendizagem em matemática, através de jogos desenvolvidos com o auxílio de ferramentas tecnológicas, tendo como foco alunos com transtorno do espectro autista. Acredita-se que promover a inclusão com o apoio de softwares que contemplam jogos se apresenta como uma relevante contribuição para o campo da educação. Espera-se também revelar o potencial das tecnologias no cotidiano das instituições de ensino, como um recurso pedagógico fundamental, merecedores de maior reconhecimento, rompendo com as metodologias tradicionais e sensibilizando profissionais envolvidos no campo da educação, especialmente os que atuam com pessoas que possuem alguma deficiência em particular. 
Apesar de investimentos em educação no Brasil, historicamente, o país apresenta problemas no campo da educação, entre eles índices elevados de evasão e repetência. Alguns resultados de avaliações nacionais e exames internacionais revelam que os alunos ao concluírem a educação básica sabem muito menos do que deveriam. A inclusão escolar também é um tema caro no país. A formação de professores é frágil ainda, em relação à preparação de profissionais para atuar com pessoas com deficiência, especialmente, alunos com transtorno do espectro autista. Esse fato evidencia que é preciso avançar na busca de estratégias inovadoras, capazes de ampliar e aprofundar a construção de conhecimentos e, consequentemente a promoção de aprendizagens. Portanto, o desenvolvimento desse projeto, está articulado ao compromisso com ações educativas, que buscam favorecer a sociedade como um todo, partindo do princípio de que a utilização de recursos didáticos baseados nas TICs, contribuem significativamente na inclusão e na formação de sujeitos autônomos.

Com a pesquisa percebe-se que a inclusão é inevitável e aponta para uma educação que oferece a todos, oportunidades mais justas perante a sociedade. Nesse sentido, o uso de jogos como recursos didáticos, podem facilitar e auxiliar no aprendizado da matemática para os alunos com TEA, desde que esse recurso seja escolhido com o propósito de aprimorar um ou mais conteúdo ministrado pelo professor, possibilitando entendimento conceitual a partir do entendimento concreto.

\section{Referencias}

Adobe Systems Incorporated. (2017). Adobe Illustrator. Disponível em: $<\mathrm{http}$ ://www.adobe.com/br/products/illustrator.html $>$. Acesso em: 10 jun. 2017.

Bittencourt, I. G. S.; Francisco, D. J. (2015). Os efeitos da produção em blog para sujeitos com Transtorno do Espectro Autista. Anais do XXVI Simpósio Brasileiro de Informática na Educação (SBIE 2015). CBIE-LACLO 2015.

Bona, B. O. (2009). Análise de softwares educativos para o ensino de Matemática nos anos iniciais do ensino fundamental. Experiências em Ensino de Ciências, Carazinho, RS, v.4, p. 35-55, maio. 2009.

Bosa, C. A. (2016). Autismo: Intervenções Psicoeducacionais. Disponível em http://dx.doi.org/10.1590/S1516-44462006000500007, Acesso em: 18 de mar. 2017.

Carvalho, M. F.; Gasparini, I.; Hounsell, M. S. (2016). Jogos Digitais Educacionais para Alfabetização Matemática: Levantamento de Habilidades e Level Design. Anais do XXVII Simpósio Brasileiro de Informática na Educação (SBIE 2016). V Congresso Brasileiro de Informática na Educação (CBIE 2016).

Corel Corporation. CorelDraw Graphics Suite. (2017). Disponível em: $<$ http://www.coreldraw.com/br/>. Acesso em: 10 jun. 2017.

Frias, E. M. A.; Menezes, M. C. B. (2008). Inclusão Escolar do Aluno com Necessidades Educacionais Especiais: contribuições ao professor no Ensino Regular. Disponível em http://www.diaadiaeducacao.pr.gov.br/portals/pde/arquivos/14628.pdf/. Acesso em: 18 de jun. 2017.

Graphic Resources S.L.. Freepik. (2017). Disponível em: <http://br.freepik.com/>. Acesso em: 01 jun. 2017.

Guiadedireito (2017). Direito a educação especial. Disponível em 
VI Congresso Brasileiro de Informática na Educação (CBIE 2017)

Anais do XXIII Workshop de Informática na Escola (WIE 2017)

http://guiadedireitos.org/index.php?

option=com_content\&view=article\&id=1075\&Itemid=285, Acesso em: 20 de jun. 2017.

Inkspace Project. Inkscape. (2017). Disponível em: <https://inkscape.org/pt-br/>. Acesso em: 07 jun. 2017.

Lemon (2017). Lemon. Disponível em: http://www.lemon.com.br/produto.cfm?id=409. Acesso em: 07 jun. 2017.

Menezes, S. V.; Roza, J. C. (2016). Genius Math: Uma Aplicação Mobile para Auxiliar a Aprendizagem da Matemática na Pré-escola. Anais do XXVII Simpósio Brasileiro de Informática na Educação (SBIE 2016). V Congresso Brasileiro de Informática na Educação (CBIE 2016)

Microsoft Corporation. (2017). Microsoft Office. Disponível em: $<$ https://www.microsoft.com/pt-br/store/b/office?icid=CNavSoftwareOffice $>$. Acesso em: 10 jun. 2017.

Minayo, M. C. S. (2010). O desafio do conhecimento: pesquisa qualitativa em saúde. 12. ed. São Paulo: Hucitec.

Passerino, L. M. (2005). Pessoas com autismo em ambientes digitais de aprendizagem: estudo dos processos de interação social e mediação. Tese de Doutorado do Programa de Pós-Graduação em Informática na Educação da Universidade Federal do Rio Grande do Sul. Disponível em http://www.lume.ufrgs.br/bitstream/handle/10183/13081/000634298.pdf? sequence $=1$. Acesso em: 23 de mai. 2017.

Sampaio, M. N.; Leite, L. S. (2004). Alfabetização tecnológica do professor. $4^{\mathrm{a}}$ ed. Petrópolis: Vozes.

Santos, W. O.; Neto, S. R. S; Junior, C. G. S; Bittencourt, I. I. (2015). Avaliação de Jogos Educativos: Uma Abordagem no Ensino de Matemática. Anais do XXVI Simpósio Brasileiro de Informática na Educação (SBIE 2015). CBIE-LACLO 2015

Seterra (2017). Sebran's ABC. Disponível em: http://online.seterra.com/pt/p/sebran. Acesso em: 07 de jun. 2017.

Sousa, F. R. M.et. al. (2012). WorldTour: Software para Suporte no Ensino para Crianças Autistas. Anais do XXIII Simpósio Brasileiro de Informática na Educação (SBIE 2012). Disponível em http://www.brie.org/pub/index.php/sbie/article/view/1806/1567. Acesso em: 24 de mar. 2017.

The Document Foundation. LibreOffice Impress. (2017). Disponível em: $<$ https://ptbr.libreoffice.org/descubra/impress/>. Acesso em: 08 jun. 2017.

Tux (2017). Tux, of Math Command. Disponível em https://tux4kids.alioth.debian.org/tuxmath/index.php. Acesso em 07 de jun. 2017. 